\title{
ENCELADUS GEODETIC FRAMEWORK
}

\author{
J. Oberst ${ }^{\text {a, }}$, H. Hussmann ${ }^{\text {a }}$, B. Giese ${ }^{\text {a, }}$ F. Sohl ${ }^{\text {a }}$, D· Shoji ${ }^{\text {b }}$, A. Stark ${ }^{\text {a }}$, K. Wickhusen ${ }^{\text {a }}$, M. Wählisch ${ }^{\text {a }}$ \\ a German Aerospace Center (DLR), Institute of Planetary Research, Berlin, Germany - \\ ${ }^{\mathrm{b}}$ Earth-Life Science Institute, Tokyo Institute of Technology, Japan \\ Juergen.Oberst@dlr.de
}

\section{Commission WG III/II}

KEY WORDS: Saturn, Cassini, Enceladus, Planetary Geodesy

\begin{abstract}
:
The small (approximately $500 \mathrm{~km}$ in diameter) satellite Enceladus is moving near the equatorial plane and deep in the gravity field of its parent planet Saturn. Owing to tidal interaction with its parent, Enceladus has adopted a pronounced 3-axial ellipsoidal shape and is tidally locked, with rotational and orbital periods of about 1.37 days. As the equator of Saturn is inclined to the planet's orbital plane, Enceladus, like most of the other satellites of Saturn, undergoes pronounced seasons. This paper gives a summary of the current status as well as shortcomings of our current knowledge regarding Enceladus' geodetic and dynamic parameters.
\end{abstract}

\section{INTRODUCTION}

The Cassini spacecraft began its tour through the Saturnian system in July 2004 and has engaged in 22 targeted flybys of Enceladus. The small satellite is moving near the equatorial plane and deep in the gravity field of Saturn. Cassini's onboard Imaging Science Subsystem (ISS) combines a high-resolution Narrow Angle Camera (NAC) (focal length: 2,000 mm) and a Wide Angle Camera (WAC) (focal length: $200 \mathrm{~mm}$ ) (Porco et al., 2004) and delivered several hundreds of images, from which control point networks and high-quality maps could be produced. By radio science data analysis, low-order gravity field parameters of Enceladus could be recovered. Regularly venting plumes of water ice and water vapour in the south-polar region revealed the presence of liquid water in the satellites' subsurface (Porco et al., 2006). Hence, Enceladus quickly moved into the focus of scientists. The preparation of future exploration will require a thorough study of all available observational data. This paper gives a summary of the current status as well as shortcomings of our current knowledge regarding Enceladus' geodetic and dynamic parameters.

\section{ENCELADUS GEODETIC AND DYNAMIC PARAMETERS}

\subsection{Orbit}

Benefitting from Cassini radio tracking and astrometric observations, the ephemerides of the satellites of Saturn could be greatly improved. The sat375 ephemeris produced at JPL (Jacobson, 2015) was used to extract orbital elements using techniques described by Stark et al., (2015). Enceladus' orbital parameters are significantly influenced by its neighboring moons, notably Dione to which it is locked in a 2:1 meanmotion resonance (Enceladus is completing two orbits about Saturn every one orbit completed by Dione). The sidereal (i.e. with respect to stars) orbital period about Saturn is 1.370218 days. The orbit is slightly inclined to Saturn's equator (Figure 1) and can be approximated by an ellipse with a small eccentricity $e=0.0049 \pm 0.0011$. Hence, the distance to Saturn's center of mass varies between 237,250 and 239,570 km (semi-major axis: $238,411 \mathrm{~km}$ ). However, as Saturn's gravity field has significant non-spherical components, the orbital ellipse precesses by
$0.33796 \pm 0.00037 \%$ with a period of $2.9164 \pm$ 0.0032 years. Consequently, the time between consecutive pericenter passages (the anomalistic period) is 2.54 minutes longer than the sidereal period. Enceladus' orbit pole precesses about Saturn's spin pole with periodicities of 2.36 and 4.98 years (Giese and Rambaux, (2015), Figure 1).

\subsection{Rotation}

2.2.1 Coordinate system definition: Solar system planets and satellites have common definitions for their coordinate systems, supported by the International Astronomical Union (e.g. Archinal et al., 2011). The origin is located at the center of mass, the $\mathrm{z}$-axis points into the direction of the spin pole (angular momentum vector), while the $\mathrm{x}$ - and $\mathrm{y}$ - axes define the equatorial plane. The $\mathrm{x}$-axis passes through the prime meridian $\left(0^{\circ}\right.$ longitude). For Enceladus, the prime meridian is fixed by the location of crater Salih, which is defined to be at $5^{\circ}$ eastern longitude (Davies and Katayama, 1983). As a result, this makes the prime meridian (and the $\mathrm{x}$-axis) to be offset from the direction to Saturn ("long axis system") by $\sim 4^{\circ}$ on average.

2.2.2 Spin pole: Enceladus' spin pole is not fixed in inertial space but is believed to track the motion of the orbit pole as shown in Figure 1. Any obliquity (angle between spin pole and orbit pole) is predicted to be smaller than $0.00045^{\circ}$ (Baland et al., 2016). The amplitude of orbit pole precession is on the order of $0.01^{\circ}$ and so is the expected amplitude of spin pole precession. Pre-Cassini spin models for Enceladus recommended by IAU (Archinal et al., 2011) had pole solutions as

$$
\left(\begin{array}{c}
D e c \\
R a
\end{array}\right)=\left(\begin{array}{l}
83.52^{\circ}-0.004^{\circ} T \\
40.66^{\circ}-0.036^{\circ} T
\end{array}\right)
$$

where $\quad T=$ time in centuries from J2000 epoch.

However, new control point calculations using high-resolution Cassini images (Giese, 2014) have fixed the mean (over 7 years) spin pole orientation at $(D e c, R a)=\left(83.54^{\circ}, 40.59^{\circ}\right)$, thus making the earlier IAU models obsolete. The new solution is close to the mean values of the orbit pole orientation (Figure 1). This supports the assumption that the spin pole is tracking the motion of the orbit pole. 

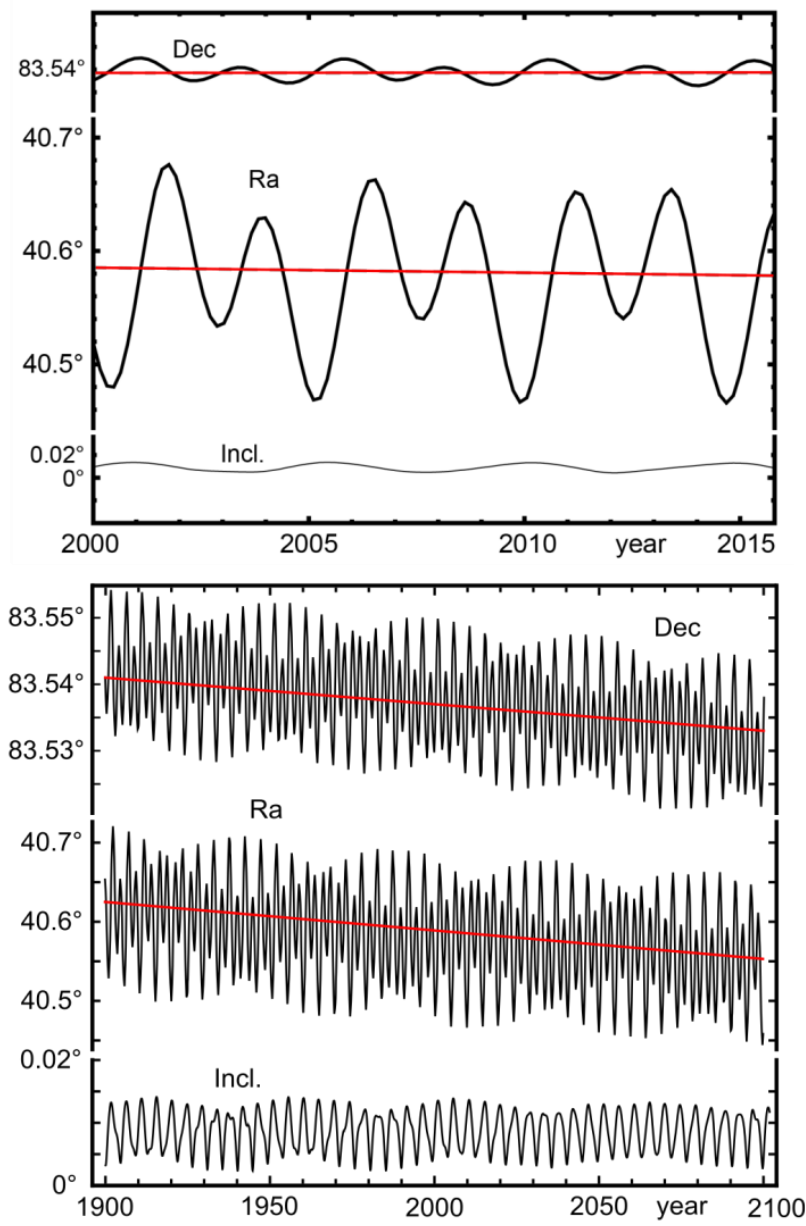

Figure 1: ICRF declination (Dec) and right ascension (Ra) of Enceladus' orbit pole determined from the ephemeris data. The top and bottom panel depict the short-term (15 years) and longterm (200 years) evolution, respectively. The orientation at the $\mathrm{J} 2000$ reference epoch is $(D e c, R a)=\left(83.538^{\circ}, 40.579^{\circ}\right)$. Red

lines indicate the orientation of Saturn's spin pole, which averages the oscillating orbit pole orientation. The lower curves show the inclination of the orbit pole to Saturn's spin pole, varying between $0.0032^{\circ}$ and $0.0139^{\circ}$ around a mean value of $0.0086^{\circ}$.

2.2.3 Rotation and longitudinal libration: Enceladus is tidally locked in synchronous rotation, i.e. the spin period is always equal to the orbital period $T_{\text {orb }}$. At uniform rotation (IAU model) the prime meridian equation is then given by (Archinal et al., 2011)

$$
W=6.32^{\circ}+\frac{360^{\circ}}{T_{\text {orb }}} d=6.32^{\circ}+262.7318996^{\circ} d
$$

where $\quad d=$ time in days from the $\mathrm{J} 2000$ epoch.

However, due to orbital perturbations and a non-spherical shape (see chapt. 2.3), Enceladus experiences forced librations in longitude, which are superimposed on the uniform rotation. There are both diurnal (1.37 days) and long-period librations (3.9 years and 11.2 years) (Rambaux et al., 2010). The presence of librations has been confirmed within control point calculations (Giese et al., 2011) with specifically diurnal librations having amplitude of $0.12^{\circ}$ (Thomas et al., 2016). By combination of 3 libration periods, the total libration amplitude shows an intricate signature over time (Figure 2).

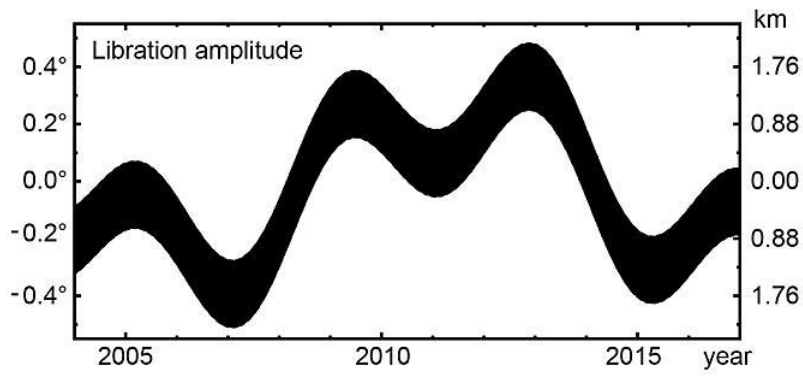

Figure 2: Total libration amplitude (on the left: in degree, on the right: respect to arc lengths along Enceladus' equator) over a full long-term cycle according to Rambaux et al. (2010). The thickness of the curve masks short-period diurnal librations with amplitude of 0.12 (Thomas et al., 2016).

\subsection{Size and Shape}

Size and shape are fundamental geodetic data for any planet or satellite. Early shape models for Enceladus were produced from Voyager flyby observations using limb-fitting techniques (Dermott and Thomas, 1994). Using Cassini data, the shape models were updated by combinations of limb-fitting and control point analysis (Table 1). In contrast to earlier findings, Enceladus was found to have a shape far from equilibrium. In particular, for Enceladus' three-axial ellipsoidal shape, $a=$ $256.2 \mathrm{~km}, b=256.2 \mathrm{~km}, c=248.6 \mathrm{~km}$, one may find ( $a-$ $c) /(b-c)=2.7$, while for hydrostatic (tidal and rotational) equilibrium this parameter should be 4.2 (McKinnon, 2015). New shape model estimates from control point analysis are currently in preparation (Zubarev, A., pers. communication).

\begin{tabular}{|l|l|l|l|l|}
\hline $\mathrm{a}[\mathrm{km}]$ & $\mathrm{b}[\mathrm{km}]$ & $\mathrm{c}[\mathrm{km}]$ & $\mathrm{R}[\mathrm{km}]$ & Ref. \\
\hline $256.3 \pm 0.3$ & $247.3 \pm 0.3$ & $244.6 \pm 0.5$ & $249.4 \pm 0.3$ & $*$ \\
\hline $256.6 \pm 0.6$ & $251.4 \pm 0.2$ & $248.3 \pm 0.2$ & $252.1 \pm 0.2$ & $* *$ \\
\hline $256.2 \pm 0.3$ & $251.4 \pm 0.2$ & $248.6 \pm 0.2$ & $252.0 \pm 0.2$ & $* * *$ \\
\hline
\end{tabular}

Table 1: Shape parameters for Enceladus. References:

* (Dermott and Thomas, 1994), ** (Thomas et al., 2007; Archinal et al., 2011), *** (Thomas et al., 2016).

\subsection{Tides}

In addition to rotational distortion, Enceladus is exposed to strong tidal forces because of its proximity to Saturn. The satellite's equilibrium shape, expressed as two tidal bulges on its near- and farside, is due to the superposition of rotation and tides (Figure 3). If the orbit of Enceladus were circular, this ellipsoidal shape would be constant. However, as the actual orbit of Enceladus is elliptical, the magnitude and the position of the tidal bulges change periodically with the cycle of Enceladus' orbital period ( $\sim 1.37$ days). In case of eccentricity tide, this dynamic displacement of the tidal bulges is caused by two mechanisms (see the details in Murray and Dermott, 1999). One is due to changing distance to Saturn, with the tidal bulges growing and decreasing according to changing tidal forces. The amplitude of the tidal displacement is estimated at $\sim 5 \mathrm{~m}$ (Hurford et al., 2007; 2009). The other mechanism is due to optical libration which causes a slight shift $\left(<0.6^{\circ}\right)$ of the tidal bulge with respect to the direction to Saturn.

By these periodical displacements of tidal bulges, changing stress patterns may be induced (Hurford et al., 2007; 2009; 2012; Smith-Konter and Pappalardo, 2008). Recent analyses show evidence for a correlation of plume activity with the tidal 
stresses on the diurnal 1.37 days cycle. Due to normal stresses water-filled cracks open down to the liquid water reservoir located at least a few $\mathrm{km}$ in the subsurface. Whereas the activity for individual jets is not synchronous with the variation of tidal stresses, the overall activity within the the south-polar terrain vary in phase with the tidal frequency (Hedman et al., 2013; Porco et al., 2014)

The dynamic displacement of the tidal bulges produces frictional heat by dissipation within the ice. In fact, Enceladus is one of the most dissipative icy satellites in our solar system. Due to conversion of the orbital energy into thermal energy, Enceladus' eccentricity is normally expected to decrease and eventually drop to zero. However, the orbital resonance with Dione maintains Enceladus' eccentricity at the current level.

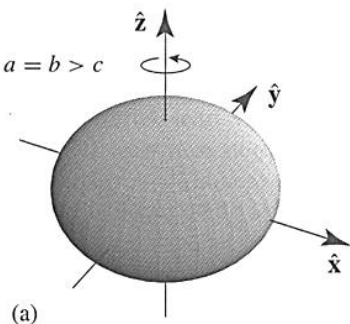

Figure 3: Examples of the equipotential surfaces arising from (a) rotational deformation, where the rotational axis is aligned with the $\mathrm{z}$ axis, and (b) tidal deformation, where the tide-raising body lies along the direction of the $\mathrm{x}$ axis (Figure adopted from Murray and Dermott, 1999).

\subsection{Mass and Gravity Field}

Radio Doppler data acquired by the Deep Space Network during the flybys of the Cassini spacecraft with Enceladus result in $G M=7.2111 \pm 0.0125 \mathrm{~km}^{3} / \mathrm{s}^{2}$ or mass $M=1.0805 \pm$ $0.0019 \times 10^{20} \mathrm{~kg}$ (Jacobson, 2015). With the radius mentioned above of $252.0 \pm 0.2 \mathrm{~km}$ (Thomas et al., 2016), this yields a mean density of $1,606 \pm 6 \mathrm{~kg} / \mathrm{m}^{3}$ and a surface gravity of $0.113 \mathrm{~m} / \mathrm{s}^{2}$

Note that Saturn's gravity at the position of Enceladus is 0.67 $\mathrm{m} / \mathrm{s}^{2}$, which is much larger (but compensated by the centrifugal force of Enceladus motion). Hence, spacecraft approaching Enceladus have to cope with strong orbital perturbations and are limited to move in so-called "quasi-satellite orbits" (Russel and Lara, 2008). The Hill sphere of Enceladus, in which Enceladus dominates gravitational motion, has a radius of only approximately $950 \mathrm{~km}$ (Spahn et al., 2006a; 2006b).

In addition, the degree 2 gravity potential has been determined from close flybys (Iess et al., 2014). The only significant nonzero terms are the zonal harmonic $J_{2}=5435.2 \pm 34.9 \times 10^{-6}$ and the sectorial harmonic $C_{22}=1549.8 \pm 15.6 \times 10^{-6}$ with a ratio $J_{2} / C_{22}=3.51 \pm 0.05$. As for the shape parameters, this ratio differs from that expected for a differentiated body in hydrostatic equilibrium (expected value: $~ 3.24$ ). The inferred moment of inertia factor is 0.335 suggesting a differentiated body. McKinnon (2015) concludes that Enceladus' gravity data are consistent with a global sub-surface ocean beneath an ice shell not thicker than $25 \mathrm{~km}$ at the South Pole.

\section{CONTROL POINT NETWORKS}

Control point networks are essentially catalogues of prominent surface features for which body-fixed coordinates are precisely known. The coordinates of the points are typically determined jointly from original measurements of point coordinates in the large numbers (blocks) of overlapping images by so-called "bundle block adjustment techniques". The control point coordinates (when available in 3-D) are an important framework for the production of shape models. Also, from the tracking of control points over time, rotational parameters of the planet or satellite may be determined.

An early control point network construction involved 38 Cassini images with resolutions ranging from 190 to 1220 meters per pixel (m/pixel) and covering a time span of 6 years. This resolution and time interval was appropriate for measuring the long-period librations shown in Figure 2. In total, 1057 image points corresponding to 186 individual ground points were measured, aiming at a dense and uniform distribution across the surface (Giese et al., 2011). More recently, new control point networks were built. The network by Thomas et al., (2016) involved 340 images and positions of 488 control points. Furthermore, A. Zubarev (pers. communication) measured 13,634 tie-point positions corresponding to 1,127 control points.

\section{MAPS}

Using the images from ISS, Enceladus image mosaics and maps, have been prepared by several teams worldwide including a complete "atlas" (resolution of 1:500,000) (Roatsch et al., 2008; 2013). Relevant map products include:

- German Aerospace Center (DLR) map and atlas (Roatsch et al., 2008; 2013)

- Lunar Planetary Institute (LPI) map (Schenk, 2014)

- Moscow State University of Geodesy and Cartography (MIIGAiK) map (Zubarev, et al., 2014)

- US Geological Survey (USGS) map (Bland et al., 2015).

New controlled global maps (image resolution: better than 1000 $\mathrm{m} /$ pixel) and a controlled South pole map (image resolution: better than $160 \mathrm{~m} /$ pixel) are being prepared (Bland, USGS, pers. communication). All maps use the surface position of the prime meridian as defined by the IAU cartography working group (Archinal et al., 2011) through the small crater Salih (Figure 4, Table 2). The Cassini imaging team proposed 64 names for prominent geological features, in addition to the 22 features already named by the Voyager team that are used in the maps [1]. By international agreement, features on Enceladus are named after people or locations in the medieval Middle Eastern literary epic "The Thousand Nights and a Night". All maps are available online $[2 ; 3 ; 4 ; 5]$.

\begin{tabular}{|l|l|l|l|}
\hline & Latitude $\left.^{\circ}{ }^{\circ}\right]$ & $\begin{array}{l}\text { Western } \\
\text { longitude }\left[{ }^{\circ}\right]\end{array}$ & Ref. \\
\hline IAU definition & & $5.0^{\circ}$ & $*$ \\
\hline $\begin{array}{l}\text { Planetary } \\
\text { Names }\end{array}$ & $5.99^{\circ}$ & $4.4^{\circ}$ & $* *$ \\
\hline DLR mosaic & $5.16^{\circ}$ & $4.27^{\circ}$ & $* * *$ \\
\hline
\end{tabular}

Table 2: Reported coordinates of crater Salih. References:

* (Archinal et al., 2011), ** [1], *** (Roatsch et al., 2008; 2013) 
The quadrangle scheme of the DLR atlas consists of 15 tiles (Figure 5), which conforms to the quadrangle scheme proposed by Greeley and Batson (1990) for larger satellites. The Enceladus atlas is available through the Cassini Imaging Team [6] and the Planetary Data System (PDS) [7].

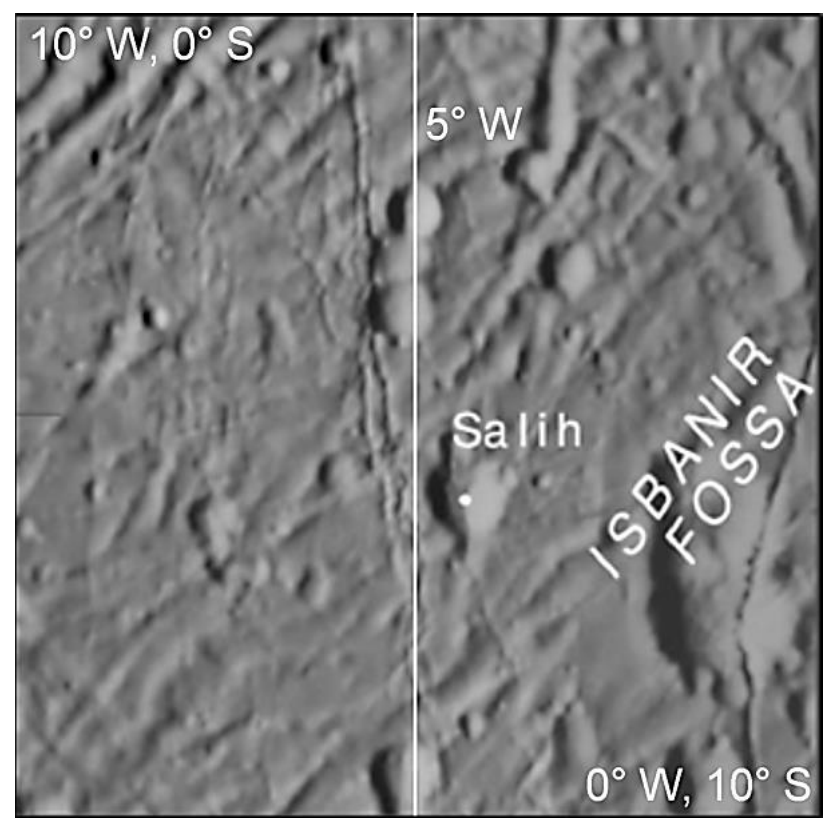

Figure 4: Crater Salih (adapted from Roatsch et al., (2008)).

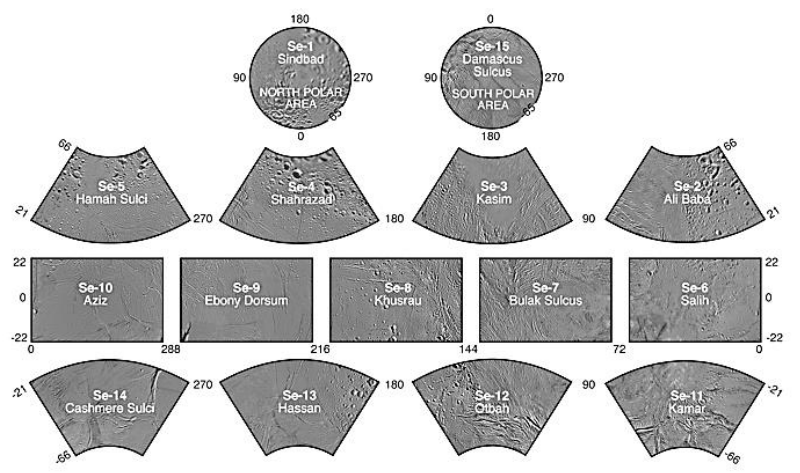

Figure 5: Quadrangle mapping scheme for Enceladus. Credit: NASA/JPL/Space Science Institute.

\section{ILLUMINATION}

With its mean semi-major axis of 9.537 AU and eccentricity of 0.054 Saturn's distance to the sun varies between 9 AU and 10.1 AU (1.35 - 1.51 bill. km). Thus the total solar flux received in orbit around Saturn varies between $17 \mathrm{~W} / \mathrm{m}^{2}$ and $13 \mathrm{~W} / \mathrm{m}^{2}(1$ $\mathrm{m}^{2}$ unit area, pointed at the sun). Saturn's equatorial plane is tilted by $26.73^{\circ}$ with respect to its orbit plane. As Enceladus' orbit inclination and obliquity are small, the moon follows the seasonal cycle of its parent planet.

Saturn will be at its next equinox position and enter southern summer season in May 2025, which will last until January 2039 (Table 3). Summer peak (southern solstice) will be in May 2032. Hence, this season will last 13.7 years. Owing to the eccentric orbit of Saturn (and equinox times not being symmetric with respect to the solar orbit period), the northern summer season will be significantly longer (15.7 years). The seasonal patterns in the illumination levels are superposed by
Saturn's changing solar distance. In November 2032, Saturn will be at its perihelion position (Table 3), which will result in maximum solar irradiation on the Southern hemisphere (peak solar power density of $\sim 17 \mathrm{~W} / \mathrm{m}^{2}$, Figure 6 ). From the illumination perspective, the years 2032 and 2033 will be favourable for a lander mission in the South Pole area, which will enjoy constant illumination, with the sun rising up to $26.7^{\circ}$ elevation during the day (orange line in the bottom diagram of Figure 7).

\begin{tabular}{|l|l|}
\hline Event & Time \\
\hline Northern Solstice & 24.May 2017 \\
\hline Saturn Aphelion & 16 April 2018 \\
\hline Equinox & 06 May 2025 \\
\hline Southern Solstice & 12. April 2032 \\
\hline Saturn Perihelion & 29 November 2032 \\
\hline Equinox & 23 January 2039 \\
\hline
\end{tabular}

Table 3: Saturn/Enceladus solar orbits and key events

The apparent size of Saturn's disk is sufficiently large to cause eclipses. Due to the rather large inclination of Saturn's equator and the orbital plane of Enceladus, eclipses are limited to time windows near equinox seasons. Eclipses occur once per orbit between 2022 - 2028 and 2036 - 2041. Eclipses will reduce total illumination by up to $8 \%$ per orbit $(2.6 \mathrm{~h} /$ orbit $)$ during equinox (see indentations of the violet and green lines in the top panel of Figure 7). As the rotation of Enceladus is locked and as Saturn always faces the same hemisphere ("nearside") of the satellite, only the nearside of Enceladus is effected by eclipse events, while on the anti-Saturn hemispheres the central body is never seen.

Note that over one full Saturn year probably no areas on Enceladus will be in permanent shadow, except for localities with extreme topography.

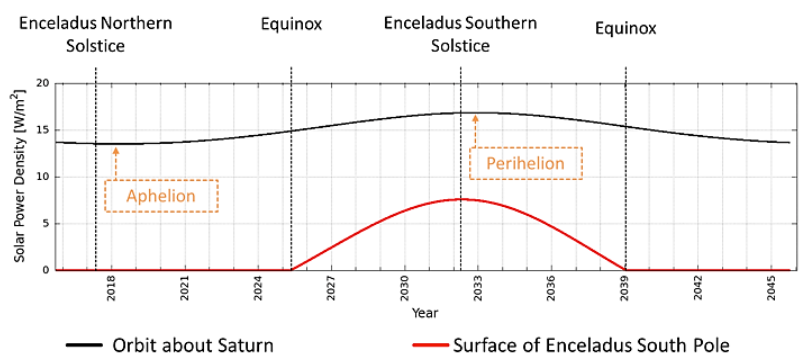

Figure 6: Solar flux for areas perpendicular to the incoming solar radiation (black) and for areas on the surface at the South Pole (red). 


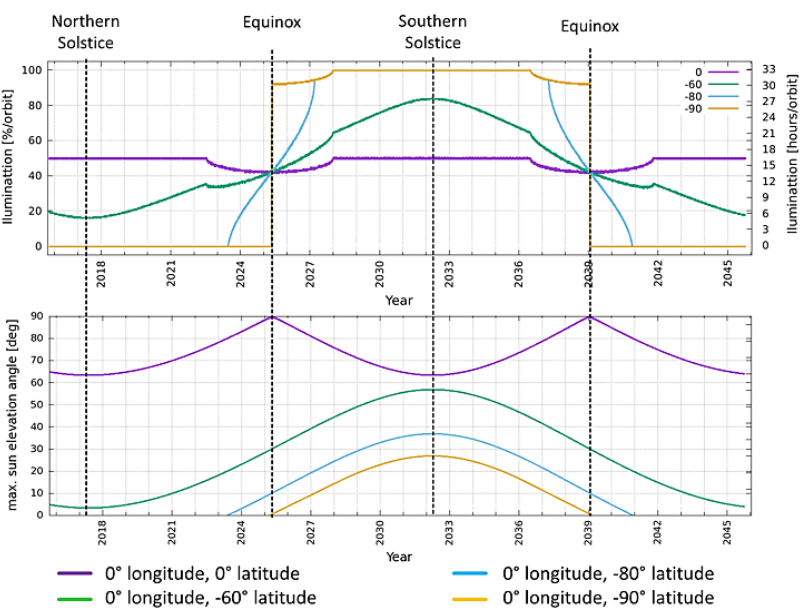

Figure 7: Illumination conditions on the prime meridian, but 4 different latitudes on the surface of Enceladus. The top diagram shows the illumination duration for each of these points in percentage per orbit and hours per orbit. The bottom diagram shows the corresponding maximum sun elevation angles for each orbit.

\section{REFERENCES}

Archinal, B. A. et al., 2011. Report of the IAU Working Group on Cartographic Coordinates and Rotational Elements: 2009. Celest Mech Dyn Astr., 109, pp. 101-135, DOI:10.1007/s10569-010-9320-4.

Bland, M. T. et al., 2015. A new Enceladus base map and global control network in support of geologic mapping. 46th Lunar and Space Science Conference \# 2303.pdf.

Greeley, R. and Batson, G., 1990. Planetary Mapping. Cambridge University Press.

Baland R.-M. et al., 2016. The obliquity of Enceladus. Icarus, 268, pp. 12-31, http://dx.doi.org/10.1016/j.icarus.2015.11.039.

Davies, M. E. and Katayama, F. Y., 1983. The Control Networks of Mimas and Enceladus. Icarus, 53, pp. 332-340, DOI:10.1016/0019-1035(83)90153-7.

Dermott, S. F. and Thomas, P. C., 1994. The determination of the mass and mean density of Enceladus from its observed shape. Icarus, 109, pp. 241-257.

Giese, B. et al., 2011. Enceladus: Evidence for librations forced by Dione. European Planetary Science Congress, EPSC Abstracts Vol. 6, EPSC-DPS2011-976.

Giese, B., 2014. An upper limit on Enceladus' obliquity. European Planetary Science Congress, EPSC Abstracts, 9, EPSC2014-419.

Giese, B. and Rambaux, N., 2015. Enceladus' long-period physical librations. European Planetary Science Congress, EPSC Abstracts, 10, EPSC2015-867.

Hedman, M. M. et al., 2013. An observed correlation between plume activity and tidal stresses on Enceladus. Nature, 500, pp. 182-184, DOI:10.1038/nature12371.
Hurford, T. A. et al., 2007. Eruptions arising from tidally controlled periodic openings of rifts on Enceladus. Nature, 447, pp. 292-294, DOI:10.1038/nature05821.

Huford, T. A. et al., 2009. Geological implications of a physical libration on Enceladus. Icarus, 203, pp. 541-552, DOI: 10.1016/j.icarus.2009.04.025

Huford, T. A. et al., 2012. Tidal control of jet eruptions on Enceladus as observed by Cassini ISS between 2005 and 2007. Icarus, 220, pp. $896-903$.

Iess, L. et al., 2014. The Gravity Field and Interior Structure of Enceladus. $\quad$ Science, 344 (6179), p. 78-80, DOI: $10.1126 /$ science. 1250551 .

Jacobson, R. A., 2015. SAT375 - JPL satellite ephemeris. (http://naif.jpl.nasa.gov/pub/naif/generic_kernels/spk/satellites/)

McKinnon, W. B., 2015. Effect of Enceladus's rapid synchronous spin on interpretation of Cassini gravity. Geophys. Res. Lett., 42, pp. 2137-2143, DOI:10.1002/2015GL063384.

Murray, C. D. and Dermott, S. F., 1999. Solar system dynamics. Cambridge University Press.

Porco C. C., et al., 2004. Cassini imaging science: instrument characteristics and anticipated scientific investigations at Saturn. Space Sci. Rev., 115, pp. 363-497.

Porco, C. C. et al., 2006. Cassini observes the active south pole of Enceladus. Science, 311, pp. 1393 - 1401.

Porco, C. C. et al., 2014. How the geysers, tidal stresses, and thermal emission across the south polar terrain of Enceladus are related. The Astron. Journal, 148: 45.

Rambaux, N. et al., 2010. Librational response of Enceladus. Geophys. Res. Lett., 37, L04202, DOI:10.1029/2009GL041465.

Roatsch, Th., et al., 2008. High-resolution Enceladus atlas derived from Cassini-ISS images. Planet. Space Sci., 56, pp. 109-116.

Roatsch, Th., et al., 2013. Recent improvements of the Saturnian satellites atlases: Mimas, Enceladus, and Dione. Planet. Space Sci., 77, pp. 118-125.

Russell, R. P. and Lara, M., 2008. On the Design of an Enceladus Science Orbit. AIAA/AAS Astrodynamics Specialist Conference, AIAA-2008-7072 Honolulu, HI, August 20.

Schenk, P. M., 2014. Planetary report, 34, no. 3, pp. 8-13.

Smith-Konter, B. and Pappalardo, R. T., 2008. Tidally driven stress accumulation and shear failure of Enceladus's tiger stripes. Icarus, 198, pp. 435-451, DOI:10.1016/j.icarus.2008.07.005.

Spahn, F. et al., 2006a. Cassini Dust Measurements at Enceladus and Implications for the Origin of the E Ring. Science, 10,311 (5766), pp. 1416-1418, DOI: $10.1126 /$ science. 1121375 .

Spahn, F. et al., 2006b. E ring dust sources: Implications from Cassini's dust measurements. Planet. Space Sci., 54, pp. 1024 1032. 
Stark, A. et al., 2015. Mercury's resonant rotation from secular orbital elements. Celest. Mech. Dyn. Astr., 123, pp. 263-277.

Thomas, P. C. et al., 2007. Shapes of the saturnian icy satellites and their significance. Icarus, 190, pp. 573-584.

Thomas, P. C. et al., 2016. Enceladus's measured physical libration requires a global subsurface ocean. Icarus, 264, pp. 37 -47 .

Zubarev, A. et al., 2014. Geodesy and cartography methods of exploration of the outer planetary systems: Galilean satellites and Enceladus. 40th COSPAR Scientific Assembly, Held 2-10 August 2014, in Moscow, Russia, Abstract B0.3-13-14.

[1] http://planetarynames.wr.usgs.gov/

[2] http://ciclops.org/view/7590/Map-of-Enceladus--December-2011

[3] http://ciclops.org/view/7591/Enceladus-Polar-Maps--December-2011

[4]http://s3.amazonaws.com/ciclops_ir_2010/6318_14983_1.pd f?AWSAccessKeyId=14V1KFXK26R9EA1V0Q02\&Expires $=1$ 498243384\&Signature $=0 \mathrm{kQNaQngiN4 \% 2BVknoIF9I9Yyz3fo}$ $\% 3 \mathrm{D}$

[5] http://www.lpi.usra.edu/icy_moons/saturn/enceladus/

[6] http://ciclops.org/maps/
[7] http://pds.jpl.nasa.gov/

(All Web references accessed 20 June 2017)

\section{ACKNOWLEDGEMENTS}

This work was supported by the German Space Agency in the context of the EnEx initiative (Figure 8).

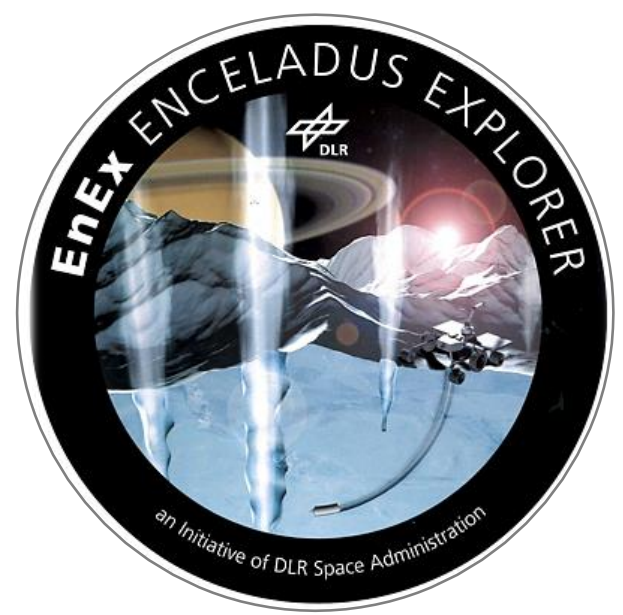

Figure 8: EnEx initiative logo. 